\title{
Points to Consider in Preventing Unfair Discrimination Based on Genetic Disease Risk: A Position Statement of the American College of Medical Genetics
}

\author{
Michael S. Watson, PhD, and Carol L. Greene, MD
}

The American College of Medical Genetics believes that fears of genetic discrimination in health insurance and employment have a negative impact on willingness to seek genetic services and to participate in genetic research. These decisions, based on fears of discrimination, could keep individuals from having services that could protect their health and that of family members by prevention and treatment of disease. Comprehensive federal legislation is needed to protect all Americans. The goal of such legislation should be to enhance the safe and effective integration of genetic services, including genetic testing, as an inseparable part of the health care system. The American College of Medical Genetics believes that:

- All Americans should be protected by genetic nondiscrimination legislation addressing both health insurance and employment.

- Protection against genetic discrimination in health insurance is needed for individuals covered by all public and private programs, whether through group or individual plans, and regardless of the mechanism by which the program is regulated.

- Protection against discrimination in employment must cover the many participants in the process including employers, employment agencies, labor organizations, and training programs, and at all steps of the employment process.

- Legislation must not impede the ability of individuals to make maximal use of genetic information in their health care and employment decision-making. It must not limit the access of health care providers to genetic information needed to ensure that the care provided is beneficial and specific to the needs of the individual.

- Like all health information, the privacy of genetic information must be adequately protected. Protection against unfair discrimination based on genetic risk for disease is achieved only by strategies that restrict use of genetic information in enrollment and rate-setting.

Dr. Watson is Executive Director of the ACMG, Bethesda, Maryland, and Dr. Greene is with Children's National Medical Center, Washington, DC.

Committee Name: Governmental and Legislative Affairs Committee.

Go to http://www.lww.com/GIM/polst.html for a printable copy of this document.
- There are significant uses of genetic technology in evaluation and management of conditions that are not familial. For example, DNA analysis is used to evaluate cancer cells for acquired mutations that can help to characterize the tumor and guide treatment. Legislation should recognize that there are different types of genetic tests, and avoid creating new barriers to use of those elements of genetic technology that do not create unique or novel risks.

- It is not easy to define "genetic test," "genetic information," and "genetic services" for legislative purposes. Key and occasionally contradictory points to consider include the following:

- Definitions must be sufficiently broad to accommodate the wide range of what is known about classical singlegene disorders and the contribution of multiple genes to common, complex diseases.

- Definitions must be sufficiently flexible to avoid becoming rapidly outdated by new developments.

- Definitions must be sufficiently narrow and clear to avoid confusion in application of statutory protection in the current system of health delivery.

- Definitions must avoid creating arbitrary distinctions that unfairly exclude some individuals from protections afforded to others.

- Protected genetic information must include that based on evaluation, testing, and family histories of individuals and their family members.

- Legislation should include enforcement to ensure compliance.

- When there is clear evidence that genetic factors contribute to an individual's vulnerability to unavoidable environmental workplace exposures, and testing is available to determine whether an individual is at increased risk, employees and potential employees should be made aware of the availability of such testing and should be fully informed of the implications of testing.

The American College of Medical Genetics believes that no American should have to choose between having a genetic test that could be important to his/her life and avoiding a genetic test to save a job or protect health insurance coverage. 
This position statement is designed primarily as an educational resource for medical geneticists and other health care providers to help them provide quality medical genetic services. Adherence to this guideline does not necessarily assure a successful medical outcome. This position statement should not be considered inclusive of all proper procedures and tests or exclusive of other procedures and tests that are reasonably directed to obtaining the same results. In determining the propriety of any specific procedure or test, the geneticist should apply his or her own professional judgment to the specific clinical circumstances presented by the individual patient or specimen. It may be prudent, however, to document in the patient's record the rationale for any significant deviation from this guideline. 